\title{
The Rotation and Variability of T Tauri Stars: Results of Two Decades of Monitoring at Van Vleck Observatory
}

\author{
W. Herbst
}

\section{Astronomy Dept., Wesleyan University, Middletown, CT 06459 USA}

\begin{abstract}
A brief history of photometric monitoring of $\mathrm{T}$ Tauri stars with a $0.6 \mathrm{~m}$ telescope at Wesleyan University is given. This is followed by discussion of three recent results: 1) the mass dependence of stellar rotation rates in the Orion Nebula Cluster, 2) a check on stellar radii estimates of pre-main sequence stars, and 3) the nature of a very peculiar object in NGC 2264.
\end{abstract}

\section{Introduction and Brief History}

T Tauri stars (TTS) are low mass $\left(\mathrm{M}<2 \mathrm{M}_{\odot}\right)$ pre-main sequence (PMS) stars with ages in the range of 0.1 to $10 \mathrm{My}$. They were originally identified as an interesting class of irregular variable stars by Joy (1942). The nature and cause of their variations remained largely unknown until the 1980's and is still mysterious in some aspects. Our photometric monitoring program, employing the $0.6 \mathrm{~m}$ (Perkin) telescope of Van Vleck Observatory, situated on the campus of Wesleyan University, began in 1981 with two principal goals. First, we wished to elucidate the nature of $T$ Tauri variables which meant observing them on a regular basis. Second, we wanted to involve students in research and train them in some methods of observational astronomy. The program began with visual observations of objects in the Orion Nebula region and quickly moved to UBVRI and $\mathrm{H} \alpha$ photoelectric photometry of bright $\mathrm{T}$ Tauri and Herbig Ae/Be (HAEBE) stars after construction of an appropriate instrument, with the help of a grant from Research Corporation. Our first papers (Herbst, Holtzmann \& Phelps 1982; Herbst, Holtzmann \& Klasky 1983; Herbst \& Stine 1984) established distinctions between what are now known as Type II (hot spot) variability and Type III (UXor) variability including discovery of the color "turnaround" at faint magnitudes which is characteristic of the latter.

In the early 1980's it was discovered that some T Tauri stars were not, in fact, irregular variables but at least some of the time showed distinctly periodic behavior (Schaefer 1983; Rydgren \& Vrba 1983). The original interpretation proposed, that we were witnessing the rotation of a spotted star, has been verified by subsequent studies and is now well established. In most cases, the spots are cooler than the photosphere, although a few examples of hot spot periodicity have been found, including one star (DN Tau) which showed hot and cool spot periodicity simultaneously at one epoch (Vrba et al. 1986). Photometric variations caused by the rotation of a star with an asymmetric distribution of cool spots on its surface was dubbed "Type I" variability by Herbst et al. (1994) 
and is now recognized as the most common cause of variations in PMS stars. Carpenter et al. (2001) estimate that $80 \%$ of the near-IR variations they detect in stars in the Orion Nebula Cluster (ONC) and surroundings can be accounted for by this mechanism. It appears to be the ONLY mechanism of variability operating in weak TTS (WTTS) - those lacking evidence for accretion disks except for an occasional flare analogous to solar flares. The best studied star exhibiting this kind of variations is V410 Tau, which was a target of intensive study at Wesleyan. We showed that the star had two large, cool, long-lived spots in opposite hemispheres which drifted in longitude relative to each other, but persisted for at least seven years and maybe longer (Vrba, Herbst \& Booth 1988; Herbst 1989). These results were basically confirmed by Doppler imaging studies in the early 1990's (Joncour, Bertout \& Menard 1994; Rice \& Streissmauer, 1996).

The recognition that it was possible to obtain rotation periods for many PMS stars by photometric monitoring led to a new class of studies which has persisted until today and continues to grow. Bouvier and collaborators applied the method to study rotation of WTTS and CTTS in associations such as Taurus using PMT's (Bouvier et al. 1986; Bouvier et al. 1993). We organized campaigns on some stars involving many observers (including some amateur astronomers) at a wide range of longitudes around the world. An early success of this program was the discovery of a rotation period for $\mathrm{T}$ Tauri itself (Herbst et al. 1986). However, it soon became clear that most of the bright CTTS and HAEBE stars were not going to yield periodicities easily, if at all. Unlike the WTTS, where dogged monitoring is usually rewarded with a period (e.g. Grankin 2001), the CTTS and HAEBE stars only rarely show significant periods (Herbst et al. 1987). Claims to the contrary by Bouvier et al. (1993) were based on a toooptimistic interpretation of noise peaks in periodograms (Herbst \& Wittenmyer 1996; Rebull 2001). When reasonably priced, off-the-shelf CCD systems became available in the late 1980's we were able to switch from PMTs to these area detectors and began focusing attention at Wesleyan on young clusters for obvious reasons of efficiency.

A pilot study of the very center of the ONC, the so-called Trapezium cluster, with our original chip, a $5 \mathrm{Kx} 5 \mathrm{~K}$ device about $1 \mathrm{~cm}$ in size, immediately yielded the first (8) rotation periods for PMS stars in the ONC (Mandel \& Herbst 1991). This was followed by a more extensive study in which we monitored 11 fields within the ONC using the small chip and discovered 35 rotation periods. Their frequency distribution was strikingly non-uniform and distinctly bimodal, with peaks near 2 days and 8 days and a clear gap at 4 days (Attridge \& Herbst 1992). Further study confirmed this result, expanding the number of rotation periods to 75. We proposed an explanation for the bimodal period distribution in terms of the "disk-locking" mechanism of Königl (1991) and Ostriker \& Shu (1995) (Choi $\&$ Herbst 1996; see also Edwards et al. 1993). It was a surprise, therefore, when Stassun et al. (1999) claimed, based on their wider field and deeper study of the ONC, that the period distribution was not bimodal. The issue was resolved by Herbst et al. (2000a) who showed that, in fact, only the brighter, more massive stars in the ONC - the ones included in the studies at VVO, which employed only the $0.6 \mathrm{~m}$ telescope - showed a bimodal distribution. Fainter, lower mass stars, which dominate the Stassun et al. (1999) sample have, in fact, quite different rotation properties as a group! The issue will be updated in the next 
section after a brief retreat from rotation issues to the general question of TTS and HAEBE star variability.

Probably the most active young star monitoring program in the world over the last decade and a half was run at Maidanak Observatory in Uzbekistan by V. Shevchenko of the Tashkent Astronomical Institute. Shevchenko and his colleagues, many of whom were students, obtained photoelectric photometry (UBVR) of hundreds of PMS stars each year using primarily $0.4 \mathrm{~m}$ telescopes. The excellent conditions at Mt. Maidanak (Ehgambendlev et al., 2000 ), including long strings of clear weather, aided this work. Supported by a grant from the Civilian Research and Development Corporation for States of the Former Soviet Union, we were able to collaborate with Shevchenko to monitor common objects at different longitudes and to make the Maidanak data easily available to the world through an ftp sight on the World Wide Web (ftp://ftp.astro.wesleyan.edu/pub/ttauri/). Analysis of these data has contributed to many papers on TTS variability, including some of our own (Herbst et al. 1994; Shevchenko \& Herbst 1998; Herbst \& Shevchenko 1999). Hopefully, the PMS monitoring programs at Mt. Maidanak will continue, led by Grankin, Ibraghimov and others, following the untimely death of Shevchenko.

\section{Updating the ONC Rotation work}

Work on rotation in the ONC, which began with Mandel \& Herbst (1991), has expanded dramatically in the last few years (Choi \& Herbst 1996; Stassun et al. 1999; Herbst et al. 2000a; Rebull 2001) spurred by new observational programs, wider field CCDs and some scientific controversy. As mentioned previously, results obtained by Stassun et al. (1999) on fainter, lower mass stars in the $\mathrm{ONC}$ and its surroundings seemed to be at odds with the primary result of Choi $\&$ Herbst, that a bimodal period distribution was present in the ONC. Herbst et al. (2000a) showed that the difference was primarily due to the different mass ranges probed in the studies - the bimodal distribution only applies to stars more massive than $0.25 \mathrm{M}_{\odot}$. Rebull (2001) surveyed a large area around the ONC (the so-called "flanking fields") and also found that the period distribution was not bimodal, although she did not have masses for most of her stars, so could not check on mass dependence issue. However, her work raises the possibility that bimodality is a feature only of the ONC itself and does not apply generally to a more heterogeneous set of PMS stars. This, in fact, is what one might expect from the disk-locking scenario since it requires rather special conditions - a set of very young stars most of which had been released from their disks only a short time (much less than $1 \mathrm{My}$ ) ago - to produce a bimodal distribution. A more heterogeneous set of disk release times would quickly eliminate bimodality from the period distribution as the stars contract rapidly and spin up conserving angular momentum on a short timescale (see Herbst et al. 2000a).

It is clearly important to consider stellar mass when discussing rotational evolution and to push the rotation studies in all clusters to the lowest possible masses. We have initiated a program in collaboration with $\mathrm{R}$. Mundt of MaxPlanck-Institute for Astronomy in Heidelberg, Germany, to study the ONC with the recently commissioned Wide Field Imager on the $2.2 \mathrm{~m}$ telescope at the European Southern Observatory in Chile. Preliminary results were reported by 


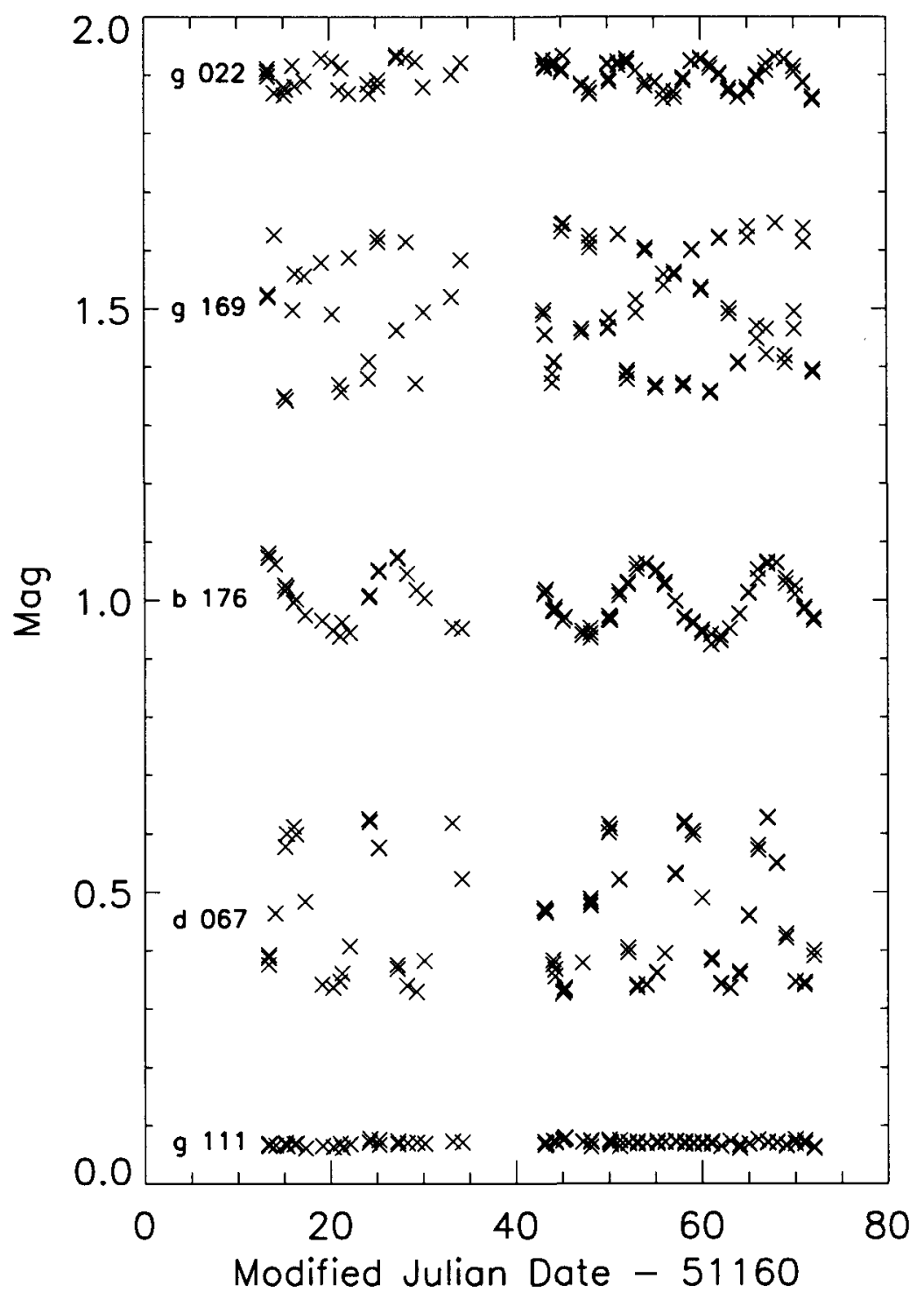

Figure 1. Some examples of periodic light curves of TTS in the ONC obtained with the $2.2 \mathrm{~m}$ telescope at the European Southern Observatory in collaboration with astronomers at the Max-Planck-Institute in Heidelberg, Germany (Herbst et al. 2000b). The bottom light curve is a non-variable star shown as a comparison object. 

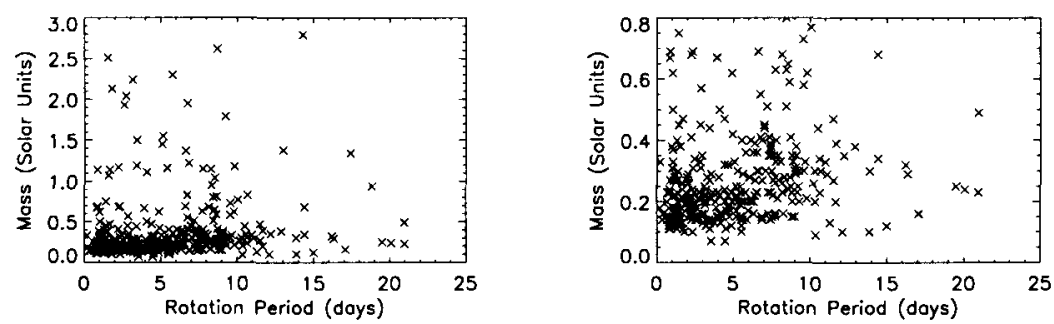

Figure 2. Rotation Period versus Mass for stars in the ONC. The left hand panel shows the full range of the data, while the right hand panel enlarges the well-populated region of mass less than $0.8 \mathrm{M}_{\odot}$.

Herbst et al. (2000b) and are briefly updated here. A full discussion is in preparation. Rotation periods were determined for more than 400 stars in the ONC. Comparison with previous studies mentioned above shows that there were 111 stars in common and 99 of these yielded identical periods. In all but one of the remaining cases, the period differences were consistent with either aliasing (with a one day observing interval) or period doubling (caused by the existence of two spots in opposite hemispheres at some epochs). This is a gratifying affirmation of the methods used to identify periodic stars and of the basic interpretation of the periodicity in terms of rotation.

A sample set of light curves for periodic stars (plus one comparison object) is shown in Figure 1. The period distribution as a function of mass is shown in Figure 2, where the left panel shows the full data set and the right panel shows an enlarged view of the important region below $0.8 \mathrm{M}_{\odot}$. A clear trend can be seen in the sense that the median rotation period decreases with stellar mass among the lower mass stars. The gap around 4 days which exists for stars more massive than $0.25 \mathrm{M}_{\odot}$ is also readily apparent. Histograms showing these features more clearly are shown by Herbst et al. (2000b).

What can it mean that, within the mass range considered here, the lower mass stars in the ONC rotate more rapidly than those of higher mass? A full discussion is not possible within the space constraints of this review. Indeed, the answer to this question is unknown at present. For the purposes of this discussion, therefore, only one speculative possibility will be mentioned. If disks control the spin-up of stars during the accretion stage then it may be that the faster rotation of the lower mass stars indicates less action by their disks in slowing the spin-up. This, in turn, may simply mean that lower mass stars have lower mass disks which cannot control stellar rotation to the same extent as their higher mass counterparts. The issue is of particular relevance to planet formation scenarios since reasonably long-lived disks of sufficient mass are required for planet formation. If low mass stars (by far the most common in the galaxy) do not have such disks, then the large majority of stars in the galaxy may not have planets. Clearly these issues require a great deal more analysis before they can be settled; our purpose here was to illustrate one important issue to which monitoring programs with small telescopes may speak. 


\section{Combining $\mathrm{v} \sin \mathrm{i}$ and period}

Another important use of rotation periods derived from synoptic studies with small telescopes is in checking a fundamental property of PMS stars - their radii. Stellar radii are essential for calculating masses and ages of PMS stars by comparison with models. Yet, the method by which radii are determined is fraught with questionable assumptions. Since there are few PMS eclipsing binaries it is usually only possible to estimate stellar radii $(R)$ from their luminosities $(L)$ and effective temperatures $\left(\mathrm{T}_{\text {eff }}\right)$, as

$$
R=\frac{L^{\frac{1}{2}}}{(4 \pi \sigma)^{\frac{1}{2}} T_{e f f}^{2}} .
$$

A fundamental assumption of this method is that the stars involved radiate isotropically. However, we know this cannot be strictly true for most $\mathrm{T}$ Tauri stars, since they exhibit optical and near-IR variations of $10-20 \%$ or more as the stars rotate - this is how their rotation periods are determined! Effective temperatures are usually assigned by comparing the spectra of these heavily magnetized, spotted and sometimes veiled TTS with spectra of normal dwarfs and giants leading to a classification on the MK system, although usually without a luminosity class. Then, a spectral type - $\mathrm{T}_{\text {eff }}$ calibration based on normal stars is invoked. There is debate in the literature about whether it is best to compare TTS with dwarfs or with giants in assigning an effective temperature, a decision which can affect the adopted $\mathrm{T}_{\text {eff }}$ by up to a few hundred degrees. Distances to the stars and extinction corrections also factor into the inferred radii through the luminosity. Clearly it would be of value to check whether results obtained by this method are reliable.

Fortunately, this can be done if one has a sufficiently large sample of stars which have rotation periods $(\mathrm{P})$ and $\mathrm{v}$ sin i measurements. Assuming a random distribution of inclination angles (i) of the rotation axes to the line of sight, one expects that,

$$
<\sin i>=\frac{\pi}{4}=\left\langle\frac{v \sin i}{v_{e q}}>\right.
$$

where $v_{e q}=2 \pi R / P$ is the equatorial rotational velocity based on a star's rotation period and radius. Rhode, Herbst \& Mathieu (2001) have recently determined $v$ sin i values for about 250 stars in the ONC, of which more than half have measured rotation periods. They find a substantial discrepancy between results obtained by these two independent methods of determining radii. The sense of the discrepancy is that the method based on effective temperatures yields radii that are systematically larger, particularly for stars of spectral class M0-M3. It is impossible to summarize here all of the possible causes and implications of this result - the reader is referred to the original paper. It is simply noted that there may well be a problem with the current method of assigning $\mathrm{T}_{\text {eff }}$ which is resulting in these stars being considered too cool by up to $600 \mathrm{~K}$ and, therefore, too low a mass by up to $60 \%$. If true, this also means that age estimates for PMS stars may be somewhat in error, complicating attempts to test various theoretical models. 


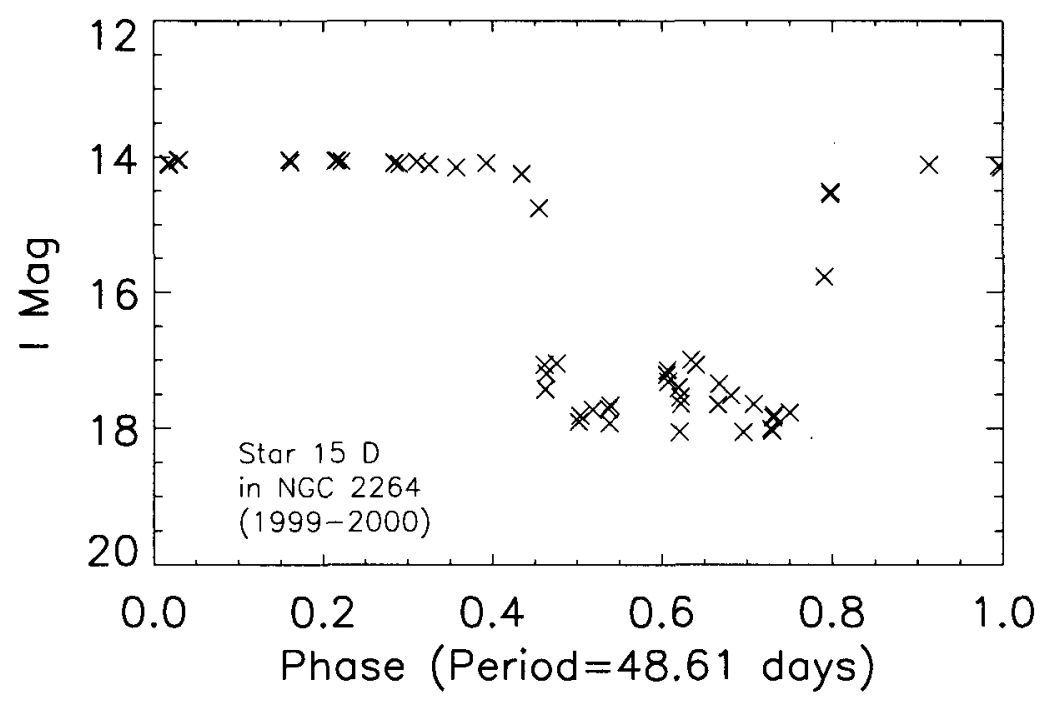

Figure 3. Light Curve of 15D. The period of 48.61 days applies only to the data obtained during the $1999 / 2000$ season.

\section{A bizarre and potentially important object}

The last point I wish to make in this review, is that monitoring programs with small telescopes may also turn up rare and potentially important objects which can be marked for intensive study using larger instruments if necessary. An example is the star known as 15D in NGC 2264 (Kearns et al. 1997; Kearns \& Herbst 1998). The light curve for this peculiar object during the most recent observing season is shown in Figure 3. It is clearly an eclipsing system with a period of 48.35 days but an extremely unusual, in fact, unique (to the author's knowledge) light curve. During eclipse, the star fades by about 3 magnitudes and stays in eclipse for about 16 days, or $1 / 3$ of its period! A recently obtained spectrum of the system during minimum light (Hamilton, 2001) indicates that the spectrum, which is typical of a $\mathrm{K} 7$ star at maximum light, is not significantly different during eclipse. Clearly, the eclipse must be caused by a large dust clump orbiting the K7 star, either on its own or attached to a secondary companion, perhaps as a circumstellar disk. In either case, the optical variations of the system during primary minimum must contain information on the density distribution of the dust clump as it passes across our line of sight. Detailed study of this system is under way and promises to reveal, for the first time, information on the distribution of dust in a circumstellar disk at much higher spatial resolution than has ever been obtained. Without dedicated monitoring programs this unique and potentially important object might not have been found for many years to come. 


\section{Final Comment on the Educational Value of this Monitoring Program}

It is sometimes argued that only robotic telescopes should be used for monitoring programs in the future because of their efficiency. I argue that, if this were to be the case, it would be a loss for science, because small telescopes and monitoring programs such as the one at Wesleyan University play an important role in education. The problem with robots for teaching is that they are remote, black boxes to students, who may have no idea about the details of where the data are coming from or how they are obtained. By contrast, when they acquire the data for themselves using semi-automated telescopes on campus, they gain a greater appreciation of what one needs to be careful about (e.g. clouds, bad seeing, proper dome alignment, stray light, the Moon, air mass effects, instrumental problems, telescope tracking or pointing problems, etc.) and how much faith to put in their data and its interpretation. If students only use robotic data, who will build the next generation of robots? I believe that the teaching function of small telescopes on campuses, which can involve students closely in actual observations should not be ignored and, in some cases, outweighs the potential efficiency gains of robotic systems. I hope that there will continue to be a place in astronomy for small telescopes engaged in monitoring programs which involve students for pedagogical purposes.

Acknowledgments. This paper is dedicated to the memory of my colleague and friend, Valery Shevchenko, who led the impressive photometric monitoring efforts of a group based at Mt. Maidanak in Uzbekistan for so many years. His untimely death at such a young age was a real loss for astronomy. I wish to thank the many collaborators who have contributed enormously to the work described here. Chief among them are Fred Vrba (USNO), Lynne Hillenbrand (CalTech), Katherine Rhode (Yale), Eric Williams (Wesleyan), Reinhard Mundt (MPIAHeidelberg), Coryn Bailer-Jones (MPIA-Heidelberg), Bob Mathieu (Wisconsin), and Mansur Ibraghimov (Ulug Bek Astron. Inst., Tashkent). This work would not have been possible without the energy, enthusiasm and talents of more than 50 Wesleyan students and Keck Northeast Astronomy Consortium summer students who have participated in the research over the years. I would particularly like to thank the following (current affiliations given in parentheses): Jon Holtzmann (NMSU), John Booth (South Pole), John Filhaber, Jody Attridge (MIT Haystack), Peter Stine (Bloomsburg), Nancy Eaton, Philip Choi (UC-Santa Cruz), Lisa Frattare (STScI), Aaron Steinhauer (Indiana), Kristin Kearns, Kathy Rhode (Yale), Gillian Curran (Wellesley), Andrew Rhodes (Wesleyan), Candice Shih (Haverford) and Arianne Donar (Wesleyan). I would also like to acknowledge the financial support over the years from many sources, including Research Corporation, the Perkin Fund, NSF, NASA, USCRDF, Sigma $\mathrm{Xi}$, Dudley Observatory, the W. M. Keck Foundation and Wesleyan University.

\section{References}

Attridge, J. A. \& Herbst, W. 1992, ApJ 398, L61

Bouvier, J., Bertout, C., Benz, W., \& Mayor, M. 1986, A\&A 165, 110 
Bouvier, J., Cabrit, S., Fernandez, M., Martin, E. L., \& Matthews, J. M. 1993, A\&A 101, 485

Carpenter, J. M., Hillenbrand, L. A. \& Skrutskie, M. F. 2001, preprint

Choi, P. I. \& Herbst, W. 1996, AJ 111, 283

Edwards, Suzan, Strom, Stephen E., Hartigan, Patrick, Strom, Karen M., Hillenbrand, Lynne A., Herbst, William, Attridge, Joanne, Merrill, K. M., Probst, Ron, Gatley, Ian 1993, AJ 106, 372

Ehgamberdiev, S. A., Baijumanov, A. K., Ilyasov, S. P., Sarazin, M., Tillayev, Y. A., Tokovinin, A. A., \& Ziad, A. 2000 A\&A 145, 293

Grankin, K. N. 2001, private communication

Hamilton, C. H. 2001, private communication.

Herbst, W. 1989, AJ 98, 2268

Herbst, W., Bailer-Jones, C. A. L., Mundt, R., Meisenheimer, K. and Wackermann, R. 2000b, A.S.P. Conf. Series, "Planetary Systems in the Universe: Observation, Formation and Evolution", A.J. Penny, P. Artymowicz, A.M. Lagrange, \& S.S. Russell, eds., in press

Herbst, W., Booth, J. F., Chugainov, P. F., Zajtseva, G. V., Barksdale, W., Covino, E., Terranegra, L., Vittone, A. \& Vrba, F. 1986, ApJ 310, L71

Herbst, W., Booth, J. F., Koret, D. L., Zajtseva, G. V., Shakhovskaya, H. I., Vrba, F. J., Covino, E., Terranegra, L., Vittone, A., Hoff, D., Kelsey, L., Lines, R., \& Barksdale, W. 1987, AJ 94, 137

Herbst, W., Herbst, D. K., Grossman, E. J. \& Weinstein, D. 1994, AJ 108, 1906 Herbst, W., Holtzman, J. A. \& Phelps, B. E. 1982, AJ 87, 1710

Herbst, W., Holtzman, J. A. \& Klasky, R. S. 1983, AJ 88, 1648

Herbst, W. \& Shevchenko, V. S. 1999, AJ 118, 1043

Herbst, W. \& Stine, P. C. 1984, AJ 89, 1716

Herbst, W., Rhode, K. L., Hillenbrand, L. A., \& Curran, G. 2000a, AJ 119, 261

Herbst, W. \& Wittenmyer, R. 1996, B.A.A.S. 189, 4908

Joncour, I., Bertout, C., Menard, F 1994, A\&A 285, L25

Joy, A. H. 1942, PASP 54, 15

Kearns, K. E., Eaton, N. L., Herbst, W. \& Mazzurco, C. J. 1997, AJ 114, 1098

Kearns, K. E. \& Herbst, W. 1998, AJ 116, 261

Königl, A. 1991, ApJ 370, 39

Mandel, G. N. \& Herbst, W. 1991, ApJ 383, L75

Ostriker, E. C. \& Shu, F. H. 1995, ApJ 447, 813

Rebull, L. M. 2001, in press

Rhode, K. L., Herbst, W. \& Mathieu, R. 2001, AJ in preparation

Rydgren, A. E. \& Vrba, F. J. 1983, ApJ 267, 191

Schaefer, B. E. 1983, ApJ 266, 458

Shevchenko, V. S. \& Herbst, W. 1998, AJ 116, 1419

Stassun, K. G., Mathieu, R. D., Mazeh, T., \& Vrba, F. J. 1999, AJ 117, 2941

Rice, J. B. \& Streissmauer, K. G. 1996, A\&A 316, 164 
Vrba, F. J., Herbst, W. \& Booth, J. F. 1988, AJ 96, 1032

Vrba, F. J., Rydgren, A. E., Chugainov, P. F., Shakovskaia, N. I., \& Zak, D. S. 1986. ApJ 306, 199 